\title{
Neuro-imagerie: que nous apprend-elle, qu'en attendre?
}

\section{Jean Martin}

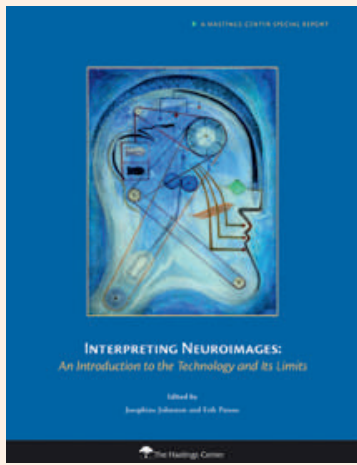

La couverture du rapport du Hastings Center sur la neuro-imagerie.
Les neurosciences et leurs contributions à l'accroissement des connaissances sont un sujet très actuel. Après une étude parue dans une revue généraliste [1], j'évoque ici un rapport du Hastings Center, institut d'éthique proche de New York, sur l'interprétation de ce que montrent les techniques de neuro-imagerie [2]. Les travaux du Hastings Center méritent toujours qu'on s'y intéresse; entre autres, j'apprécie beaucoup l'approche des problèmes de Erik Parens [3]. Quelques extraits.

D'abord un caveat: «Ce que nous voyons par des neuro-images à propos d'une fonction psychologique donnée ne peut être solide que si notre connaissance de cette fonction ou manifestation est elle-même solide». Ainsi, Helen Mayberg et ses collègues pensaient avoir découvert une «signature neurale» du phénotype dépression, avant de prendre en compte que ce tableau clinique n'est pas simple et que, pour le moins, il y a lieu d'étudier plusieurs types de dépression (ce qu'à vrai dire les cliniciens savent de longue date me semble-t-il). Plus loin: «Quelle est la précision de la corrélation entre ce qu'un scan montre de la réaction d'un sujet à la vision de visages effrayants ou plutôt neutres et la fonction physiologique 'phénotypique' - que nous appelons anxiété?»

Comme il fut un temps où l'espoir des généticiens était d'identifier le gène «unique responsable» d'une certaine maladie, celui des neuroscientifiques a pu être de découvrir la zone spécifique ou le circuit responsable pour un trouble donné. Des décennies plus tard, on sait que les relations univoques de ce type sont l'exception et pas la règle (une exception connue étant la maladie de Huntington). Il est probable qu'on peut arriver à la même manifestation "phénotypique » par différents chemins neuronaux.

Est important le point de vue systémique, de l'individu comme être bio-psycho-social: «Il est essentiel de passer de l'analyse au niveau d'une partie du cerveau à l'analyse du cerveau globalement, cerveau qui fonctionne dans un corps qui lui-même est dans un contexte social». Des allers et retours entre les niveaux d'analyse «local» (micro) et global (macro) sont nécessaires pour progresser dans l'explication des relations entre fonctionnement cérébral objectivable et ressenti psychologique.

Parens évoque la grande question du libre arbitre. Nous somme fascinés, dit-il, par l'idée que ces images permettent de s'approcher de notre ressenti de nousmêmes comme «moi», comme humain vivant en ce moment. Un des plus importants de ces ressentis étant notre sensation d'être libre, et un autre la réalisation de la mesure dans laquelle nous ne sommes pas libres. Surprenante remarque: «L'énigme au cœur de l'histoire d'Adam et Eve est précisément qu'ils ont fait leur choix librement et que, par ailleurs, dans le contexte biblique, ils n'auraient pas pu faire autrement».

A vrai dire, dit Parens, nous devons probablement abandonner le fantasme qu'une ligne donnée de recherche pourra résoudre ces énigmes. Peut-être devons-nous plutôt chercher la meilleure manière de vivre avec elles? Il est possible que la neuro-imagerie fournisse des éléments selon quoi, dans telle circonstance, telle personne ne pouvait pas choisir un autre comportement; la plupart du temps cependant, nous devrons évaluer la tension entre les vues fournies par différentes lentilles/lunettes pour interpréter la même action. Nous nous approcherons mieux de la réalité en «oscillant» entre une vision de nos actions comme déterminées de l'extérieur et une vision qu'elles sont librement choisies.

Il conclut que leur rapport [2] «soutient une voie moyenne, à propos de neuro-imagerie, entre l'optimisme extravagant et le pessimisme extravagant». En cherchant à décrire les promesses et les limites de ces technologies dans la compréhension des comportements normaux ou anormaux. «Employées avec discernement en conjonction avec les instruments d'autres sciences (psychologie, philosophie, droit et d'autres formes de savoir), elles apportent des éclairages sur les capacités complexes qui constituent nos esprits (minds), et nous aident à mieux comprendre, et vivre avec, l'énigme du caractère libre ou surdéterminé de nos pensées et actions.»

Well... Sommes-nous très avancés par un tel travail sophistiqué d'analyse et de réflexion? Certains penseront qu'il y a là du «tout est dans tout et inversement»... ou qu'il s'agit de déductions de simple bon sens, à la portée de chacun. Dans tous les cas, il reste important de disposer d'études qui rassemblent les données pertinentes et les confrontent entre elles, faisant le tri entre acquis vérifiés et prétentions excessives; s'efforçant de faire la synthèse des connaissances à un moment donné de nos histoires scientifique et sociétale.

1 Martin J. Questions autour des neurosciences et de leur pouvoir explicatif. Bull Méd Suisses. 2014;95(18):720-1.

2 Johnston J, Parens E. Interpreting Neuroimages: an introduction to the technology and its limits. A Special Report of the Hastings Center. March-April 2014.

3 Parens E. Afterword - Living with the ancient puzzle. In: Johnston J, Parens E. op. cit., p. 50-2. 